the most frequent infection among respiratory tract infections (30.1\%).

Conclusion Since, recent studies regarding to infectious diseases could be used for national planning and medical requirements' preparation, it seems that periodical assessment of these diseases is mandatory.

\section{P0-0181 ACUTE BRUCELLOSIS: CLINICAL PRESENTATIONS AND COMPLICATIONS IN CHILDREN}

${ }^{1}$ A Elhassanien, ${ }^{2} \mathrm{M}$ Alazmi. ${ }^{1}$ Pediatrics, Mansoura Faculty of Medicine, Mansoura, Egypt; ${ }^{2}$ Pediatrics, Aladan Hospital, Kuwait, Kuwait

\subsection{6/archdischild-2014-307384.842}

Brucellosis is a systemic infectious zoonotic disease andit is still an important public health problem in Arabian Peninsula. The clinical presentation of brucellosis is non-specific, and the infection varies in its course and severity.

Objective To evaluate common presentations and complications of involvement of acute Brucella infection in children presented to the paediatric emergency unit of Aladanhospital

Methods Design: Retrospective study

Setting: Paediatric emergency unit and paediatric department of Aladdin hospital, Kuwait between April 2008 and April 2013.

Subjects: Sixty two children with acute brucellosis

The diagnosis of brucellosis was made with compatible clinical findings, positive Brucella agglutination 1/160 titers, and/or the isolation of Brucella species. Complication was defined as the presence of symptoms or physical signs of infection at a particular anatomic site in a patient with active brucellosis. Data of the patients were reviewed in the medical records specially clinical presentation, complications, laboratory results, and treatment given.

Results Out of 62 patients, 36(58.1\%) were male and 26 $(41.9 \%)$ were female. The mean age was $9.5 \pm 3.2$ years. Arthritis (42 patients, $67.7 \%$ ) was the most frequent, presentation followed by fever without source (24 patients, 38.7\%), and gastrointestinal system (11 patients, 17.7\%). The diagnosis depended on increase of brucella titer more than $1 / 160$ in 57 patients (91.9\%). Source of infection in patients was mostly ingestion of unpasteurized milk.

Conclusion Brucellosisis still an important health problem in Kuwait and must be taken inconsideration in children presented with fever without a source or arthritis.

\section{PO-0182 DIAGNOSTIC IMPORTANCE OF SERUM CRP AND IL-6 FOR EARLY INFANTILE SEPSIS}

${ }^{1}$ A Elhassanien, ${ }^{2} \mathrm{M}$ Alazmi, ${ }^{3} \mathrm{H}$ Alghaiaty. ${ }^{1}$ Pediatrics, Mansoura Faculty of Medicine, Mansoura, Egypt; ${ }^{2}$ Pediatrics, Aladan Hospital, Kuwait, Kuwait; ${ }^{3}$ Pediatrics, Benha University Faculty of Medicine, Benha, Egypt

\subsection{6/archdischild-2014-307384.843}

Objectives To assess the efficacy of early estimation of serum high-sensitivity C-reactive protein (hs-CRP) and interleukin (IL)6 to differentiation between infants less than 3 months of age had sepsis and those free of infection and to assess their use as early predictor for result of blood culture (BC).
Patients and methods The study included 90 infants less than 3 month old, admitted to paediatric department of Benha university hospital, all of them showed clinically features of sepsis. Our patients were subdivided into; clinically septic infants with positive BC, clinically septic infants with negative BC and suspected septic infants with negative BC. Two venous blood samples were obtained: The first at time of hospital admission for ELISA estimation of hs-CRP and IL- 6 serum levels and the second sample was obtained either at time of development of clinical signs of sepsis or at $72 \mathrm{~h}$ in non-infected groups.

Results Blood culture was positive in 42 infants, 21 infants were clinically infected but with negative $\mathrm{BC}$ and 27 infants were suspected to have sepsis and BC were negative. Serum hs-CRP and IL-6 levels were significantly higher in infants with positive BC compared with those with negative BC. Assessing the predictive factors for infantile sepsis with positive $\mathrm{BC}$ by regression analysis showed that high total WBC count, high hs-CRP, and serum IL6 were the most significant predictors.

Conclusion Early estimation of serum hs-CRP andIL-6 levels could provide an early prediction for positive BC so allowing early initiation of therapy.

\section{PO-0183 ROLE OF ORAL CORTICOSTEROIDS BEFORE ADMISSION IN INFANTS WITH ACUTE MODERATELY ILL BRONCHIOLITIS}

${ }^{1} \mathrm{JC}$ Flores-Gonzalez, ${ }^{1} \mathrm{P}$ Comino-Vazquez, ${ }^{1} \mathrm{~B}$ Serrano-Moyano, ${ }^{1} \mathrm{E}$ Palma-Zambrana, ${ }^{1} B$ Grujic, ${ }^{1} \mathrm{~A}$ Estalella-Mendoza, ${ }^{1} \mathrm{IM}$ Calvo-Morales, ' ${ }^{1} \mathrm{~S}$ Garofano-Montero, ${ }^{1} \mathrm{P}$ RodriguezCampoy, ${ }^{1} \mathrm{JJ}$ Perez-Guerrero, ${ }^{1} \mathrm{RM}$ Garcia-Ortega, 'L Garcia-Garcia, ${ }^{1} \mathrm{FJ}$ Dávila-Corrales, ${ }^{2}$ AM Lechuga-Sancho. ${ }^{1}$ Pediatrics Department, Hospital Universitario Puerta Del Mar, Cádiz, Spain; ${ }^{2}$ Cadiz University, Hospital Universitario Puerta Del Mar, Cádiz, Spain

\subsection{6/archdischild-2014-307384.844}

Background and aims Current evidence does not support a clinically relevant effect of systemic glucocorticoids in reducing length of hospitalisation (LOS) in acute bronchiolitis. To evaluate whether treatment with oral corticosteroids prior to admission (CO) decreases LOS in patients admitted for acute moderate bronchiolitis (MB).

Material and methods In a context of a randomised, controlled, double-blind clinical trial, 185 patients with MB were included and grouped in receiving $\mathrm{CO}$ before admission for more than 24 h or not. Patients who received corticosteroids during hospitalisation and patients with risk factors for severe bronchiolitis were excluded. LOS as the main variable was recorded. Secondary: clinical scale, respiratory rate and oxygen saturation on admission.

Results 26.5\% received corticosteroids before to admission. Demographic and clinical data were similar $(\mathrm{p}>0.05)$ : Males (47\% vs $51 \%$ ), exclusively breastfeeding (51\% vs $56 \%$ ), or RSV positive (61\% vs 60\%), parental smoking (45\% vs $39 \%)$ and atopy (38\% vs $28 \%)$. There were statistically significant differences in mean age (3.5 vs 1.7 months, $\mathrm{p}=0.000$ ), severity clinical scale $(5,37$ vs 4.93 points, $\mathrm{p}=0.021)$ and in receiving salbutamol $(85.7 \%$ vs $19.1 \%, p=0.000)$. There was no difference in respiratory rate $(\mathrm{p}=0.584)$ or oxygen saturation on admission $(\mathrm{p}=0.07)$. The LOS was 2.43 vs 3.22 days, $\mathrm{p}=0.004$.

Conclusions In our series, $\mathrm{CO}$ administration before admission to BA decreases LOS significantly. The CO group was older but more severe; both can be a confusion factor. 